OPEN ACCESS

Edited by:

James Lawrence Wynn,

University of Florida, USA

Reviewed by:

Ronald James Wong,

Stanford University, USA

Paul Fisch,

University Medical Center Freiburg,

Germany

*Correspondence:

Shengyong Mao

maoshengyong@163.com

Specialty section:

This article was submitted to

Microbial Immunology,

a section of the journal

Frontiers in Microbiology

Received: 27 December 2016

Accepted: 28 February 2017

Published: 14 March 2017

Citation:

Liu J, Bian G, Sun D, Zhu W and

Mao S (2017) Starter Feeding

Supplementation Alters Colonic

Mucosal Bacterial Communities and

Modulates Mucosal Immune

Homeostasis in Newborn Lambs.

Front. Microbiol. 8:429

doi: $10.3389 /$ fmicb.2017.00429

\section{Starter Feeding Supplementation Alters Colonic Mucosal Bacterial Communities and Modulates Mucosal Immune Homeostasis in Newborn Lambs}

\author{
Junhua Liu ${ }^{1}$, Gaorui Bian ${ }^{2}$, Daming Sun ${ }^{1}$, Weiyun Zhu ${ }^{1}$ and Shengyong Mao ${ }^{1 *}$ \\ 1 Jiangsu Key Laboratory of Gastrointestinal Nutrition and Animal Health, Laboratory of Gastrointestinal Microbiology, College \\ of Animal Science and Technology, Nanjing Agricultural University, Nanjing, China, ${ }^{2}$ Department of NGS Sequencing, Tianyi \\ Health Sciences Institute, Zhenjiang, China
}

This study aims to investigate the effect of starter feeding supplementation on colonic mucosal bacterial communities and on mucosal immune homeostasis in pre-weaned lambs. We selected eight pairs of 10-day-old lamb twins. One twin was fed breast milk (M, $n=8)$, while the other was fed breast milk plus starter $(M+S, n=8)$. The lambs were sacrificed at 56 days age. Colonic content was collected to determine the $\mathrm{pH}$ and the concentrations of volatile fatty acids (VFA) and lactate. The colonic mucosa was harvested to characterize the bacterial communities using Illumina MiSeq sequencing and to determine mRNA expression levels of cytokines and toll-like receptors (TLR) using quantitative real-time PCR. The results show that starter feeding decreased luminal $\mathrm{pH}$ and increased the concentrations of acetate, propionate, butyrate, total VFA, and lactate in the colon. The principal coordinate analysis (PCA) and analysis of molecular variance show that starter feeding supplementation significantly affected the colonic mucosal bacterial communities with a higher relative abundance of the dominant taxa unclassified S24-7, Oscillibacter, Prevotella, Parabacteroides, Bifidobacterium, Ruminobacter, and Succinivibrio, and a lower proportion of unclassified Ruminococcaceae, RC9_gut_group, Blautia, Phocaeicola, Phascolarctobacterium, unclassified BS11_gut_group, unclassified family_XIII, and Campylobacter in lambs. Meanwhile, starter feeding decreased mRNA expression of TLR4 and cytokines TNF- $\alpha$ and IFN- $\gamma$ in colonic tissue. Furthermore, the changes in the colonic mucosal mRNA expression of TLR and cytokines were associated with changes in mucosal bacterial composition. These findings may provide new insights into colonic mucosal bacteria and immune homeostasis in developing lambs.

Keywords: starter feeding, colonic mucosa, bacterial community, immune homeostasis, lamb

\section{INTRODUCTION}

Gastrointestinal microbiota are integral to feed digestion, nutrient absorption and metabolism, immune response, and gastrointestinal development in ruminants (Yáñez-Ruiz et al., 2015). The gastrointestinal microbiome can be manipulated by nutritional interventions to improve productivity and health. However, the complexity and resilience of the ecosystem in adult 
ruminants can preclude such alterations (Yáñez-Ruiz et al., 2015). Recent findings have indicated that early life, when there is unstable and fragile gastrointestinal microbial ecology, is an advantageous time to intervene and change the developmental profile of the gastrointestinal microbiota and impact adult health and performance (Abecia et al., 2013; Yáñez-Ruiz et al., 2015). Therefore, implementing nutritional interventions to affect gastrointestinal microbiota at an early age can improve lifelong health and performance in ruminants and other animals.

One such nutritional intervention used is supplementation of breast milk feeding with a concentrate starter in ruminants, which enhances gastrointestinal fermentation and promotes overall gastrointestinal development (Jiao et al., 2015a; Wang et al., 2016). Previous studies have demonstrated that compared with milk feeding only, concentrate starter feeding helps shape and diversify ruminal microbial composition in calves (Malmuthuge et al., 2013) and goat kids (Jiao et al., 2015a). Jiao et al. (2016) found that concentrate feeding decreased bacterial diversity in the colonic digesta of goat kids. Furthermore, Malmuthuge et al. (2014) reported a difference in the bacterial communities of colonic digesta and mucosa in preweaned calves, suggesting that colonic mucosal bacteria may serve some specific functions, e.g., host metabolism and immune response, in young ruminants. However, little information is available regarding the effect of starter feeding on the colonic mucosal bacterial community in young ruminants, despite the importance of this bacteria in animal health. Thus, more attention should be paid to the effects of starter feeding on the colonic mucosal bacterial community in preweaned ruminants.

Colonic mucosal microbiota are integral to host immune maturation. Toll-like receptors (TLR), as novel receptors mediating innate immune responses, can recognize microbiota and their products (Abreu, 2010). Recent studies have demonstrated that changes in ruminal epithelial bacterial diversity and some specific commensal microbes is associated with changes in the expression of TLR during high-concentrate diet feeding in steers (Chen et al., 2012) and goats (Liu et al., 2015). Furthermore, microbiota and their products bind to TLR and may subsequently initiate proinflammatory pathways (Abreu, 2010). Thus, understanding the impact of starter feeding supplementation on the gene expression of TLR and cytokines as well as the role of mucosal microbiota in host immune maturation in young ruminants is necessary for their health and performance in adulthood. In the present study, we hypothesized that concentrate starter feeding changes the colonic mucosal bacterial community, and that these alterations can modulate the immune response in lambs. Our first objective was to investigate the effect of starter feeding supplementation on the colonic mucosal bacterial community and expression of TLR and cytokines in preweaned lambs. Our second objective was to evaluate the relationship between the bacterial community and host immune response in the colonic mucosa of lambs.

\section{MATERIALS AND METHODS}

\section{Animal Experimental Design}

The experimental design and procedures were approved by the Animal Care and Use Committee of Nanjing Agricultural
University. The experiment was carried out using Suzhou $\mathrm{Hu}$ sheep at a breeding farm in the Jiangsu province, China. Eight pairs of healthy, 10-day-old lamb twins ( $\mathrm{Hu}$ sheep, a native Chinese sheep breed) were selected. One kid from each pair remained with the mother and received milk ad libitum without receiving starter feed ( $\mathrm{M}$ group, $n=8$ ), while the other kid was separated from the mother and received starter feed $(\mathrm{M}+\mathrm{S}$ group, $n=8$ ) from 4:00 a.m. to 7:00 p.m. every day in a separate pen. During this period, lambs in the $M+S$ group were fed milk for $1 \mathrm{~h}$ at each fixed time point (6:30 a.m., 10:30 a.m., and 3:30 p.m.). When the dry matter intake (DMI) of the lambs' starter reached $200 \mathrm{~g} /$ animal $^{-1} \mathrm{~d}^{-1}$, the amount of starter did not rise any further. The eight lambs in the $\mathrm{M}+\mathrm{S}$ group maintained a $200 \mathrm{~g} /$ animal $^{-1} \mathrm{~d}^{-1}$ starter intake for an average of 14 days before sacrifice. All lambs received oat hay ( $10.05 \%$ crude protein, $28.71 \%$ crude fiber) and water ad libitum. The ewes were fed three times per day according to the farm's feeding management schedule. None of the lambs in the $\mathrm{M}$ and $\mathrm{M}+\mathrm{S}$ groups had access to the ewes' feed. The DMI of the starter in the $\mathrm{M}+\mathrm{S}$ group was recorded every day, and the body weights of each lamb was measured weekly (before morning feeding). The experimental starter diets were designed according to the nutrient requirements of $\mathrm{Hu}$ sheep lambs (NY/T8162004; Ministry of Agriculture of China, 2004). The nutrient composition of the starter diet is presented in Table $\mathbf{1}$ (Liu et al., 2017).

\section{Sample Collection}

Lambs were stunned by captive bolt and exsanguination at 56 days of age. A representative sample of colon digesta was collected from the proximal colon immediately after slaughter to determine the $\mathrm{pH}$ value. Colon digesta from each lamb were homogenized and mixed thoroughly with twice the amount of distilled water. The mixtures were then immediately centrifuged at $12,000 \times \mathrm{g}$, and the supernatants were stored at $-20^{\circ} \mathrm{C}$ until analysis for volatile fatty acids (VFA) and lactic acid. Within 5 min, a segment of the colon tissue was harvested and immediately washed three times in ice-cold, phosphate-buffered saline. A

TABLE 1 | Ingredient and chemical composition of the starter diet (DM ${ }^{a}$ basis).

\begin{tabular}{lrlr}
\hline Ingredient & \% DM & Component & \\
\hline Maize starch & 51.60 & DM, \% & 88.78 \\
Soybean meal & 28.00 & Crude protein, \% DM & 25.15 \\
Corn gluten meal & 15.00 & Crude fat, \% DM & 3.80 \\
Soybean oil & 1.20 & Crude ash, \% DM & 6.33 \\
Limestone meal & 0.80 & Crude fiber, \% DM & 6.34 \\
CaHPO4 & 1.80 & Starch, \% DM & 45.92 \\
Salt & 0.60 & Metabolic energy & , MJ/kg DM \\
Premix & 1.00 & & 11.43 \\
\end{tabular}

${ }^{a} \mathrm{DM}$, dry matter.

${ }^{b}$ Contained $16 \%$ calcium carbonate, $102 \mathrm{~g} / \mathrm{kg}$ of $\mathrm{Zn}, 47 \mathrm{~g} / \mathrm{kg}$ of $\mathrm{Mn}, 26 \mathrm{~g} / \mathrm{kg}$ of Cu, 1,140 $\mathrm{mg} / \mathrm{kg}$ of I, $500 \mathrm{mg} / \mathrm{kg}$ of Se, $340 \mathrm{mg} / \mathrm{kg}$ of Co, $17,167,380 \mathrm{IJ} / \mathrm{kg}$ of vitamin A, 858,370 $\mathrm{IU} / \mathrm{kg}$ of vitamin $D$, and $23,605 \mathrm{IU} / \mathrm{kg}$ of vitamin $E$.

${ }^{c}$ Calculated value based on database of the nutrient requirement for lamb (NY/Y816-2004; Ministry of Agriculture of China, 2004). 
portion of the tissue sample was cut into smaller pieces $(\sim 0.5 \times$ $0.5 \mathrm{~cm}$ ) and immediately frozen in liquid nitrogen for RNA extraction. Another portion of the tissue sample was cut to $\sim 1$ $\times 1 \mathrm{~cm}$ and scraped from the underlying tissue using a germ-free glass slide, immediately transferred into liquid nitrogen, and then stored at $-80^{\circ} \mathrm{C}$ until microbial DNA extraction. A final portion was immediately fixed in $4 \%$ paraformaldehyde (Sigma, St. Louis, MO, USA) and $2.5 \%$ glutaraldehyde for histomorphometric microscopy analysis.

\section{Physiological Parameter Measurements}

A portable $\mathrm{pH}$ meter (HI 9024C; HANNA Instruments, Woonsocket, RI, USA) was used to determine the $\mathrm{pH}$ of colonic digesta. Capillary column gas chromatography (GC-14B, Shimadzu, Japan; Capillary Column: $30 \mathrm{~m} \times 0.32 \times 0.25 \mathrm{~mm}$ film thickness; Column temperature $=110^{\circ} \mathrm{C}$, injector temperature $=$ $180^{\circ} \mathrm{C}$, detector temperature $=180^{\circ} \mathrm{C}$ ) was used to measure VFA concentration (Qin, 1982). Lactate concentration was detected using a method described by Barker and Summerson (1941).

\section{Microbial DNA Isolation}

One gram of colonic mucosal tissue was used for DNA extraction. The DNA was extracted by a PowerSoil DNA Isolation Kit (MOBIO Laboratories, Carlsbad, CA, USA, catalog 12888-100). The solution was precipitated with ethanol, and the pellets were suspended in a $50-\mu \mathrm{L}$ Tris-EDTA buffer. DNA was quantified using PicoGreen dsDNA reagent kit (Invitrogen Ltd., Paisley, UK) with a Molecular Devices SpectraMax Microplate Reader (Molecular Devices, Sunnyvale, CA, USA).

\section{PCR Amplification, Illumina MiSeq Sequencing, and Sequencing Data Processing}

The V4 regions of bacterial 16S rRNA genes were amplified by PCR (Initial denaturation at $95^{\circ} \mathrm{C}$ for $2 \mathrm{~min}, 25$ cycles of denaturation at $95^{\circ} \mathrm{C}$ for $1 \mathrm{~min}$, annealing at $55^{\circ} \mathrm{C}$ for $1 \mathrm{~min}$, elongation at $72^{\circ} \mathrm{C}$ for $1 \mathrm{~min}$, and final extension at $72^{\circ} \mathrm{C}$ for $\left.5 \mathrm{~min}\right)$ using primers $515 \mathrm{~F}\left(5^{\prime}-\right.$ barcode-GTGCCAGCMGCCGCGGTAA-3 ${ }^{\prime}$ ) and 806R (5'-barcode-GGACTACHVGGGTWTCTAAT- $3^{\prime}$ ). Amplicons were purified using the Qiagen QIAquick PCR purification kit (Qiagen, Duesseldorf, Germany) according to the manufacturer's instructions and quantified using PicoGreen dsDNA reagent kit (Invitrogen, Paisley, UK). Purified amplicons were pooled in equimolar, and the amplicon size was determined by Aglient 2200 Bioanalyzer (Agilent Technologies, CA, USA). The pooled product was pair-end sequenced $(2 \times 300)$ on an Illumina MiSeq platform according to standard protocols.

For data analyses, raw Illumina fastq files were demultiplexed, quality filtered, and analyzed using Quantitative Insights into Microbial Ecology (QIIME, v.1.8.0), as described by Caporaso et al. (2010b) and with the following criteria, as described by Mao et al. (2015): Operational taxonomic units (OTU) were clustered with a 97\% similarity cut-off using UPARSE (Edgar, 2013), and chimeric sequences were identified and removed using UCHIME (Edgar et al., 2011). The most abundant sequences within each OTU (representative sequences) were aligned to the
Greengenes database using PyNAST (Caporaso et al., 2010a) with the default parameters set by QIIME. Taxonomy was assigned to representative sequences using QIIME (Wang et al., 2007) with a confidence value of 0.8 against the Greengenes $16 \mathrm{~S}$ rRNA gene dataset (v.13.8) (DeSantis et al., 2006). Rarefaction curves and alpha and beta diversity calculations were also performed using QIIME. Principal coordinate analysis (PCA) was used to compare groups of samples based on unweighted UniFrac distance metrics (Lozupone and Knight, 2005), and an unweighted distancebased analysis of molecular variance (AMOVA) was conducted to assess significant differences among samples using the MOTHUR v.1.3.9 program (Schloss et al., 2009).

\section{Histological Measurements}

The colonic tissues were embedded in paraffin, sectioned into $6 \mu \mathrm{m}$, and stained with hematoxylin and eosin ( $\mathrm{H} \& \mathrm{E})$. During histomorphometric analyses, the microscopist was blinded to treatment conditions. For each lamb, two slides were prepared and two images were captured per slide, resulting in a total of 32 replicates per measurement per group. Predefined criteria described by Steele et al. (2011) were used to assess colonic injury using Image Pro Plus software (Media Cybernetics, Bethesda, MD, USA). The criteria were as follows: a score of one indicated no lesions or minor lesions; a score of five indicated minor lesions with mucosa sloughing; and a score of nine indicated severe, deep lesions with large amounts of mucosa sloughing.

The tissues were fixed with $2.5 \%$ glutaraldehyde for at least $24 \mathrm{~h}$, postfixed in $1 \%$ osmium, and embedded in Epon araldite. A glass knife was used to cut semithin sections $(0.25-0.5 \mu \mathrm{m})$ and ultrathin sections $(70-90 \mathrm{~nm})$. To stain semithin sections, $1 \%$ toluidine blue and $1 \%$ sodium borate were used, while uranyl acetate and lead citrate were used to stain ultrathin sections. A transmission electron microscope (H-7650; Hitachi Technologies, Tokyo, Japan) was used to examine and determine ultrastructures of the colonic tissue.

\section{RNA Extraction and Quantitative Real-Time PCR (qRT-PCR)}

Total RNA was extracted from the colonic tissue using TRIzol (Takara Bio, Otsu, Japan), as described by Chomczynski and Sacchi (1987). RNA concentrations were then quantified using a NanoDrop spectrophotometer (ND-1000UV-Vis; Thermo Fisher Scientific, Waltham, MA, USA). The absorption ratio (260/280 $\mathrm{nm}$ ) of all of the samples was between 1.8 and 2.0, indicating high RNA purity. Aliquots of RNA samples were subjected to electrophoresis through a $1.4 \%$ agarose-formaldehyde gel to verify integrity. The concentration of RNA was adjusted to 1 $\mu \mathrm{g} / \mu \mathrm{L}$ based on optical density and stored at $-80^{\circ} \mathrm{C}$. Total RNA $(1 \mu \mathrm{g})$ was reverse-transcribed using a PrimeScript RT Reagent Kit with gDNA Eraser (Takara Bio, Otsu, Japan) according to the manufacturer's instructions.

The primers of cytokine (Liu et al., 2013), TLR (Charavaryamath et al., 2011), and glyceraldehyde-3-phosphate dehydrogenase (GAPDH; Wang et al., 2009) genes used in the present study were described in previous studies. All of the primer sequences are listed in Table S1. The primers were synthesized by Invitrogen Life Technologies (Shanghai, China). 
The ABI 7300 Real-time PCR System (Applied Biosystems, Foster City, CA, USA) with SYBR green dye fluorescence detection was used to perform qRT-PCR of the target genes and GAPDH. Amplification conditions were as follows: $95^{\circ} \mathrm{C}$ for $30 \mathrm{~s}$ followed by 40 cycles of $5 \mathrm{~s}$ at $95^{\circ} \mathrm{C}$ and $31 \mathrm{~s}$ at $57.5^{\circ} \mathrm{C}$ (for GAPDH) or $62^{\circ} \mathrm{C}$ (for the cytokines and TLR). Each sample contained 1-10 ng cDNA in $2 \times$ SYBR Green PCR Master Mix (Takara Bio, Otsu, Japan) and $200 \mathrm{nmol} / \mathrm{L}$ of each primer in a final volume of 20 $\mu \mathrm{L}$. All measurements were performed in triplicate. The negative controls were a reverse-transcription-negative blank of each sample and a no-template blank. The GAPDH (a housekeeping gene) mRNA level was used to normalize the relative amount of each studied mRNA, and the $2^{-\Delta \Delta C T}$ method was used to analyze the data (Livak and Schmittgen, 2001).

\section{Statistical Analyses}

Statistical analyses were performed using the SPSS software package (SPSS v.16, SPSS Inc.). The normality of the distribution of variables was assessed with the Shapiro-Wilk test. The data found to have a normal distribution were analyzed by the Independent Samples $t$-test procedure, according to the following model: $[Y=u+C+e$ ], where $u$ is the mean, $C$ is the effect of diet, and $e$ is the residual error. The Kruskal-Wallis test was used to analyze variables found to have a non-normal distribution according to the following statistical model: $H=$ $\frac{12}{n(n+1)} \sum_{i=1}^{k} \frac{R i^{2}}{n_{i}}-3(n+1)$, where $H$ is the Kruskal-Wallis test, $n$ is the number of measurements, $R_{i}$ is the sum of the ranks, and $n_{i}$ is the number of experiments. Significance was declared at $P<0.05$.

Correlation analysis was assessed by Spearman's correlation test using GraphPad Prism v.5 (GraphPad Software, San Diego, CA, USA). Significance was declared at $P<0.01$.

\section{RESULTS}

\section{Animal}

We observed no significant differences in birth weights $(3.43 \pm$ 0.10 vs. $3.31 \pm 0.07 \mathrm{~kg}, P=0.375)$ and final body weights at 56 days age (14.89 \pm 0.36 vs. $14.44 \pm 0.34 \mathrm{~kg}, P=0.381)$ between the $\mathrm{M}$ and $\mathrm{M}+\mathrm{S}$ groups. During the feeding trial, the average total $\mathrm{DMI}$ of starter per lamb in the $\mathrm{M}+\mathrm{S}$ group was $5.54 \pm 0.16 \mathrm{~kg}$.

\section{pH, VFA, and Lactate Concentrations in Colonic Contents}

As shown in Table 2, compared with milk-fed lambs, starterfed lambs had a higher concentration of total VFA $(P=0.001)$, acetate $(P=0.018)$, propionate $(P<0.001)$, butyrate $(P<$ $0.001)$, and lactate $(P<0.001)$, but had lower luminal $\mathrm{pH}(P$ $=0.002)$ and acetate to propionate ratio $(P<0.001)$ in colonic content. Starter feeding did not affect other VFA concentrations significantly $(P=0.485)$.

\section{Characterization of the Colonic Mucosal Bacterial Communities}

After quality control, 697,630 valid reads were obtained in all samples with an average of 43,602 sequences per sample. MOTHUR analysis showed that 7,752 OTU at sequence
TABLE 2 | The effect of starter feeding on colonic fermentation in lambs at the time of slaughter ${ }^{a}$.

\begin{tabular}{|c|c|c|c|}
\hline Item & $M^{b}$ & $\mathrm{M}+\mathrm{S}^{\mathrm{c}}$ & $P$-value \\
\hline $\mathrm{pH}$ & $6.99 \pm 0.08$ & $6.76 \pm 0.15$ & 0.002 \\
\hline Total VFA ${ }^{d}, \mu \mathrm{mol} / \mathrm{g}$ & $48.30 \pm 5.38$ & $62.55 \pm 7.18$ & 0.001 \\
\hline Acetate, $\mu \mathrm{mol} / \mathrm{g}$ & $35.72 \pm 3.44$ & $41.90 \pm 5.54$ & 0.018 \\
\hline Propionate, $\mu \mathrm{mol} / \mathrm{g}$ & $7.63 \pm 0.82$ & $12.14 \pm 2.33$ & $<0.001$ \\
\hline Butyrate, $\mu \mathrm{mol} / \mathrm{g}$ & $2.81 \pm 1.10$ & $6.09 \pm 1.17$ & $<0.001$ \\
\hline Others $^{\mathrm{e}}, \mu \mathrm{mol} / \mathrm{g}$ & $2.15 \pm 0.69$ & $2.42 \pm 0.83$ & 0.485 \\
\hline Acetate: Propionate & $4.71 \pm 0.44$ & $3.54 \pm 0.69$ & 0.001 \\
\hline Lactate, $\mu \mathrm{mol} / \mathrm{g}$ & $1.85 \pm 0.19$ & $2.47 \pm 0.25$ & $<0.001$ \\
\hline
\end{tabular}

a Values are means $\pm S D, n=8$.

${ }^{b} M$, milk.

${ }^{c} M+S$, milk plus starter.

${ }^{d}$ VFA, volatile fatty acid.

${ }^{e}$ Others, valerate+isobutyrate+isovalerate.

divergences of 0.03 were classified based on these valid sequences. The average number of OTU was $485 \pm 6$, with an average coverage of $99.77 \pm 0.01 \%$. The Chaol richness, abundance-based coverage estimator (ACE), and Shannon and simpson diversity indices were $580 \pm 9,573 \pm 6,4.30$ \pm 0.07 , and $0.04 \pm 0.01$, respectively. We found a total of 18 phyla in all samples. The most dominant phyla were Firmicutes (48.58\%) and Bacteroidetes (36.33\%), and the next dominant phyla were Proteobacteria (4.00\%), Verrucomicrobia (3.91\%), and Actinobacteria (1.28\%). Unclassified bacteria (3.22\%) together with these five phyla represented $97.32 \%$ of total reads. The proportion of the phyla Tenericutes, Planctomycetes, Lentisphaerae, Spirochaetae, Cyanobacteria, Fusobacteria, and Fibrobacteres accounted for $<1.00 \%$ of total sequences. We did not detect the phyla Candidate, Elusimicrobia, Synergistetes, Deferribacteres, and Chloroflexi in all of the samples. We found a total of 218 taxa (at the genus level) in all samples. The dominant bacterial taxa were unclassified Ruminococcaceae (18.20\%), Bacteroides (9.96\%), unclassified S24-7 (7.84\%), and unclassified Lachnospiraceae (6.93\%), followed by unclassified Christensenellaceae (5.23\%), unclassified Bacteroidales (3.88\%), Akkermansia (3.87\%), RC9_gut_group (3.67\%), Alistipes (3.66\%), unclassified Clostridiales (2.73\%), Blautia (2.62\%), Oscillibacter (2.49\%), Phocaeicola (1.86\%), Prevotella (1.33\%), Phascolarctobacterium (1.33\%), and unclassified Defluviitaleaceae $(1.16 \%)$. The proportion of other taxa was below $1.00 \%$ of total sequences. As was shown in Figure S1, the top 50 bacterial taxa of different samples were presented in the heat map.

\section{Effect of Starter Feeding on Colonic Mucosal Bacterial Diversity}

The rarefaction curves of colonic mucosal bacterial communities (Figure S2, at dissimilarity levels of 0.03) showed that all curves approached a plateau, suggesting that deeper sequencing was not responsible for an increase of OTU across all samples. We used the unweighted UniFrac metric in MOTHUR to evaluate $\beta$-diversity across the samples (Figure 1). As shown in the PCA figure, the plots of the $\mathrm{M}$ and $\mathrm{M}+\mathrm{S}$ groups were distinctly 


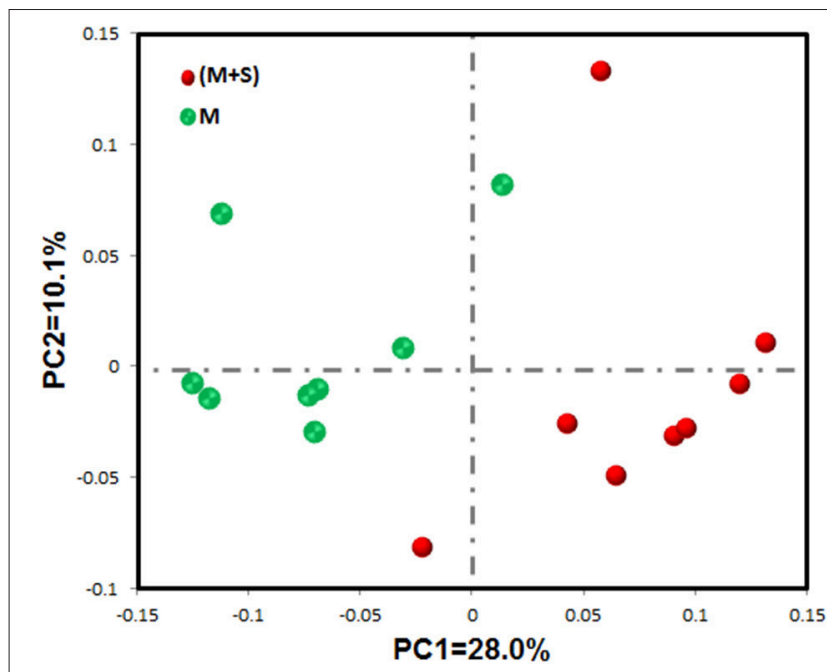

FIGURE 1 | Differences in colonic mucosal bacterial structures between the $\mathbf{M}$ and $\mathbf{M}+\mathbf{S}$ groups. Unweighted UniFrac principal coordinate analysis (PCA) of colonic mucosal microbiota was based on the operational taxonomic unit (OTU) data. The marks relate to donor lambs of different groups: M group (O) and M+S group (०)

separated (Figure 1; axis $1+$ axis $2=38.1 \%$ ). The AMOVA analysis shows that starter feeding significantly affected the colonic mucosal bacterial communities (AMOVA, $F s=3.68$, $P=0.001)$. The effects of starter feeding on the $\alpha$-diversity of colonic mucosal bacterial communities are shown in Table 3. The results show that starter feeding supplementation increased Chaol richness $(P=0.034)$, and no significant difference in OTU numbers $(P=0.203)$, ACE $(P=0.181)$, and Shannon $(P=0.373)$ or simpson $(P=0.331)$ indices.

\section{Effect of Starter Feeding on the Relative Abundance of Colonic Mucosal Bacteria}

At the phylum level (Table 4), we found that compared with the $M$ group, the $M+S$ group had a higher relative abundance of Bacteroidetes $(P=0.027)$ and Actinobacteria $(P=0.005)$, with a lower relative abundance of Firmicutes $(P=0.027)$, unclassified Bacteria $(P=0.046)$, and Cyanobacteria $(P=0.036)$. We observed no significant difference in the proportions of the phyla Proteobacteria $(P=0.600)$, Verrucomicrobia $(P=$ $0.462)$, Tenericutes $(P=0.172)$, Planctomycetes $(P=0.141)$, Lentisphaerae $(P=0.294)$, Spirochaetae $(P=0.916)$, and Fusobacteria $(P=0.916)$ between the $M$ and $M+S$ groups.

At the genus level (Table 5 and Figure S3), starter feeding caused an increase in the relative abundance of the dominant taxa unclassified S24-7 $(P=0.002)$, Oscillibacter $(P=$ 0.046), Prevotella $(P=0.009)$, Parabacteroides $(P=0.002)$, Bifidobacterium $(P=0.002)$, Ruminobacter $(P=0.002)$, and Succinivibrio $(P=0.006)$. Starter feeding also caused a decrease in the relative abundance of unclassified Ruminococcaceae $(P=0.006)$, RC9_gut_group $(P=0.027)$, Blautia $(P=$ 0.002), Phocaeicola $(P=0.036)$, Phascolarctobacterium $(P=$ 0.009), unclassified BS11_gut_group $(P=0.027)$, unclassified
TABLE 3 | Effects of starter feeding on the diversity of colonic mucosal bacterial communities at the $3 \%$ dissimilarity levela ${ }^{\mathrm{a}}$.

\begin{tabular}{lccccc}
\hline & OTUb & ACE & Chao 1 value & Shannon index & Simpson \\
\hline$M$ & $477 \pm 24$ & $565 \pm 28$ & $562 \pm 41$ & $4.36 \pm 0.16$ & $0.03 \pm 0.01$ \\
$M+S$ & $492 \pm 22$ & $582 \pm 20$ & $598 \pm 13$ & $4.23 \pm 0.38$ & $0.04 \pm 0.04$ \\
$P$-value & 0.203 & 0.181 & 0.034 & 0.373 & 0.331 \\
\hline
\end{tabular}

a Values shown are means $\pm S D, n=8$

${ }^{b}$ OTU, operational taxonomic units.

${ }^{c} A C E$, abundance-based coverage estimator.

TABLE 4 | The effect of starter feeding on relative abundance of phylum level ( $\%$ of total sequences) in colonic mucosa ${ }^{a}$.

\begin{tabular}{lccc}
\hline Phylum & $\mathbf{M}$ & $\mathbf{M}+\mathbf{S}$ & $\boldsymbol{P}$-value \\
\hline Firmicutes & $53.11 \pm 5.61$ & $44.04 \pm 8.81$ & 0.027 \\
Bacteroidetes & $31.35 \pm 5.31$ & $41.31 \pm 10.02$ & 0.027 \\
Proteobacteria & $3.86 \pm 1.07$ & $4.14 \pm 2.33$ & 0.600 \\
Verrucomicrobia & $4.11 \pm 1.54$ & $3.71 \pm 3.05$ & 0.462 \\
Unclassified Bacteria & $3.71 \pm 0.55$ & $2.72 \pm 1.36$ & 0.046 \\
Actinobacteria & $0.62 \pm 0.27$ & $1.93 \pm 1.23$ & 0.005 \\
Tenericutes & $1.03 \pm 0.50$ & $0.68 \pm 0.55$ & 0.172 \\
Planctomycetes & $0.61 \pm 0.21$ & $0.43 \pm 0.39$ & 0.141 \\
Lentisphaerae & $0.55 \pm 0.58$ & $0.27 \pm 0.24$ & 0.294 \\
Spirochaetae & $0.37 \pm 0.28$ & $0.40 \pm 0.37$ & 0.916 \\
Cyanobacteria & $0.44 \pm 0.34$ & $0.20 \pm 0.15$ & 0.036 \\
Fusobacteria & $0.16 \pm 0.13$ & $0.14 \pm 0.11$ & 0.916 \\
Others & $0.09 \pm 0.06$ & $0.02 \pm 0.01$ & 0.001 \\
\hline
\end{tabular}

a Values are means $\pm S D, n=8$.

family_XIII $(P=0.016)$, Campylobacter $(P=0.016)$, unclassified Firmicutes $(P=0.002)$, Pseudobutyrivibrio $(P=0.009)$, Barnesiella $(P=0.046)$, Lactobacillus $(P=0.001)$, unclassified Gastranaerophilales $(P=0.036)$, Butyrivibrio $(P=0.006), d g A$ 11 gut_group $(P=0.001)$, and Dorea $(P=0.012)$.

\section{Morphology and Ultrastructure of Colon Tissues}

For the lambs from the $\mathrm{M}$ group, we observed sloughing of the mucosal surface in the representative cross-sections of the colonic tissue (Figure 2A). For the lambs from the $\mathrm{M}+\mathrm{S}$ group, we observed that the intercryptal surface was covered by an irregular layer of mucus (Figure 2B). The colonic injury scores of lambs in the $\mathrm{M}+\mathrm{S}$ group were significantly lower than that of lambs in the M group $(2.04 \pm 0.16$ vs. $4.63 \pm 0.25, P<0.001)$. The lambs from the $M$ group had sparse and irregular microvilli in the ultrastructure of the colon tissue (Figure 2C), while the lambs from the $\mathrm{M}+\mathrm{S}$ group had clear and organized microvillus clusters (Figure 2D). For the lambs in the $\mathrm{M}$ group, intercellular tight junction erosion, and mitochondrial swelling appeared in the cells (Figure 2E); for the lambs in the $\mathrm{M}+\mathrm{S}$ group, we observed normal and clear cell organelles and tight junction bands in the cells (Figure 2F). 
TABLE 5 | Effects of starter feeding on average relative abundance of genus level (\% of total sequences) in colon mucosa, ranked by alphabetical order of first letter of phylum, family, and genus name.

\begin{tabular}{|c|c|c|c|c|c|}
\hline \multirow[t]{2}{*}{ Phylum } & \multirow[t]{2}{*}{ Family } & \multirow[t]{2}{*}{ Genus } & \multicolumn{2}{|c|}{ Abundance (\%) } & \multirow[t]{2}{*}{$P$-value } \\
\hline & & & $\mathbf{M}$ & $\mathbf{M}+\mathbf{S}$ & \\
\hline Actinobacteria & Bifidobacteriaceae & Bifidobacterium & $0.31 \pm 0.17$ & $0.95 \pm 0.70$ & 0.002 \\
\hline \multirow[t]{8}{*}{ Bacteroidetes } & BS11_gut_group & Unclassified BS11_gut_group & $1.73 \pm 1.64$ & $0.16 \pm 0.25$ & 0.027 \\
\hline & Porphyromonadaceae & Parabacteroides & $0.20 \pm 0.09$ & $1.70 \pm 1.39$ & 0.002 \\
\hline & & Barnesiella & $0.65 \pm 0.80$ & $0.18 \pm 0.26$ & 0.046 \\
\hline & Prevotellaceae & Prevotella & $0.25 \pm 0.25$ & $2.41 \pm 3.08$ & 0.009 \\
\hline & Rikenellaceae & dgA-11_gut_group & $0.57 \pm 0.39$ & $0.01 \pm 0.02$ & 0.001 \\
\hline & & RC9_gut_group & $5.01 \pm 1.59$ & $2.32 \pm 3.21$ & 0.027 \\
\hline & S24-7 & Unclassified S24-7 & $0.60 \pm 0.65$ & $15.07 \pm 14.06$ & 0.002 \\
\hline & Unclassified Bacteroidales & Phocaeicola & $3.40 \pm 3.15$ & $0.33 \pm 0.39$ & 0.036 \\
\hline Cyanobacteria & Unclassified Gastranaerophilales & Unclassified Gastranaerophilales & $0.44 \pm 0.34$ & $0.20 \pm 0.15$ & 0.036 \\
\hline \multirow[t]{10}{*}{ Firmicutes } & Family_XIII & Unclassified Family_XIII & $0.80 \pm 0.25$ & $0.50 \pm 0.33$ & 0.016 \\
\hline & Lachnospiraceae & Blautia & $3.92 \pm 1.63$ & $1.31 \pm 0.60$ & 0.002 \\
\hline & & Butyrivibrio & $0.35 \pm 0.04$ & $0.23 \pm 0.07$ & 0.006 \\
\hline & & Dorea & $0.33 \pm 0.11$ & $0.21 \pm 0.05$ & 0.012 \\
\hline & & Pseudobutyrivibrio & $0.61 \pm 0.50$ & $0.22 \pm 0.29$ & 0.009 \\
\hline & Lactobacillaceae & Lactobacillus & $0.71 \pm 0.48$ & $0.05 \pm 0.05$ & 0.001 \\
\hline & Oscillospiraceae & Oscillibacter & $1.70 \pm 0.90$ & $3.28 \pm 1.74$ & 0.046 \\
\hline & Ruminococcaceae & Unclassified Ruminococcaceae & $21.76 \pm 2.63$ & $14.65 \pm 4.90$ & 0.006 \\
\hline & Unclassified Firmicutes & Unclassified Firmicutes & $0.77 \pm 0.31$ & $0.19 \pm 0.23$ & 0.002 \\
\hline & Veillonellaceae & Phascolarctobacterium & $1.91 \pm 0.64$ & $0.75 \pm 0.63$ & 0.009 \\
\hline \multirow[t]{3}{*}{ Proteobacteria } & Campylobacteraceae & Campylobacter & $0.93 \pm 0.46$ & $0.32 \pm 0.26$ & 0.016 \\
\hline & Succinivibrionaceae & Ruminobacter & $0.01 \pm 0.00$ & $1.08 \pm 2.43$ & 0.002 \\
\hline & & Succinivibrio & $0.04 \pm 0.04$ & $0.61 \pm 0.60$ & 0.006 \\
\hline
\end{tabular}

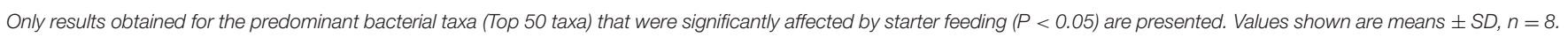

\section{Changes in mRNA Expression of Cytokines and TLR with Starter Feeding Supplementation in the Colonic Mucosa}

As shown in Figure 3, starter feeding decreased the mRNA expression of cytokines TNF- $\alpha(P<0.001)$ and IFN- $\gamma(P<$ $0.001)$ in the colonic mucosa. We found no significant differences in mRNA expression of IL-1 $\beta(P=0.759)$, IL-6 $(P=0.472)$, IL-10 $(P=0.068)$, and IL-12 $(P=0.986)$ between the $\mathrm{M}$ and $\mathrm{M}+\mathrm{S}$ groups. Meanwhile, starter feeding also decreased colonic mucosal TLR4 $(P=0.017)$ mRNA expression, and we observed no significant differences in mRNA expression of TLR2 $(P=$ $0.251)$, TLR3 $(P=0.938)$, and TLR5 $(P=0.223)$.

\section{Correlation Analyses}

Figure 4 depicts the relationships between the relative abundance of colonic mucosal bacteria and TLR and cytokine expression. The relative mRNA expression of IL- 6 was negatively associated with the relative abundance of the genus Blautia $(P=0.0076)$, while IL-10 mRNA expression was negatively linked with the relative proportion of the genus Phocaeicola $(P=0.0079)$. IL12 mRNA expression was positively correlated with the relative abundance of the genus Alistipes $(P=0.0057)$, whereas the mRNA expression level of TNF- $\alpha$ was positively associated with the relative abundance of the taxa unclassified Ruminococcaceae $(P=0.0022), d g A-11$ gut_group $(P=0.0003)$, Blautia $(P$ $<0.0001)$, Lactobacillus $(P<0.0001)$, Dorea $(P=0.0076)$, unclassified Firmicutes $(P=0.0012)$, and Butyrivibrio $(P=$ $0.0017)$, and negatively correlated with the abundance of the taxa unclassified S24-7 $(P=0.0019)$, Prevotella $(P=0.0056)$, Parabacteroides $(P=0.0014)$, Ruminobacter $(P=0.0027)$, and Bifidobacterium $(P=0.0077)$. IFN $-\gamma$ mRNA expression was positively correlated with the relative proportion of the taxa $d g A$ 11_gut_group $(P=0.0049)$, Blautia $(P=0.0005)$, Lactobacillus $(P=0.0001)$, Phocaeicola $(P=0.0099)$, Pseudobutyrivibrio $(P$ $=0.0054)$, Desulfovibrio $(P=0.0095)$, unclassified Firmicutes $(P$ $<0.0001)$, Butyrivibrio $(P=0.0012)$, and Phascolarctobacterium $(P=0.0034)$, and negatively associated with the abundance of the taxa unclassified S24-7 $(P=0.0005)$, Parabacteroides $(P=$ $0.0008)$, Ruminobacter $(P=0.0059)$, and Bifidobacterium $(P=$ $0.0074)$. The mRNA expression of TLR4 was positively associated with the taxa unclassified Ruminococcaceae $(P=0.0075)$, Pseudobutyrivibrio $(P=0.0037)$, and unclassified Firmicutes $(P$ $=0.0093$ ), and negatively linked with the taxa unclassified S24-7 


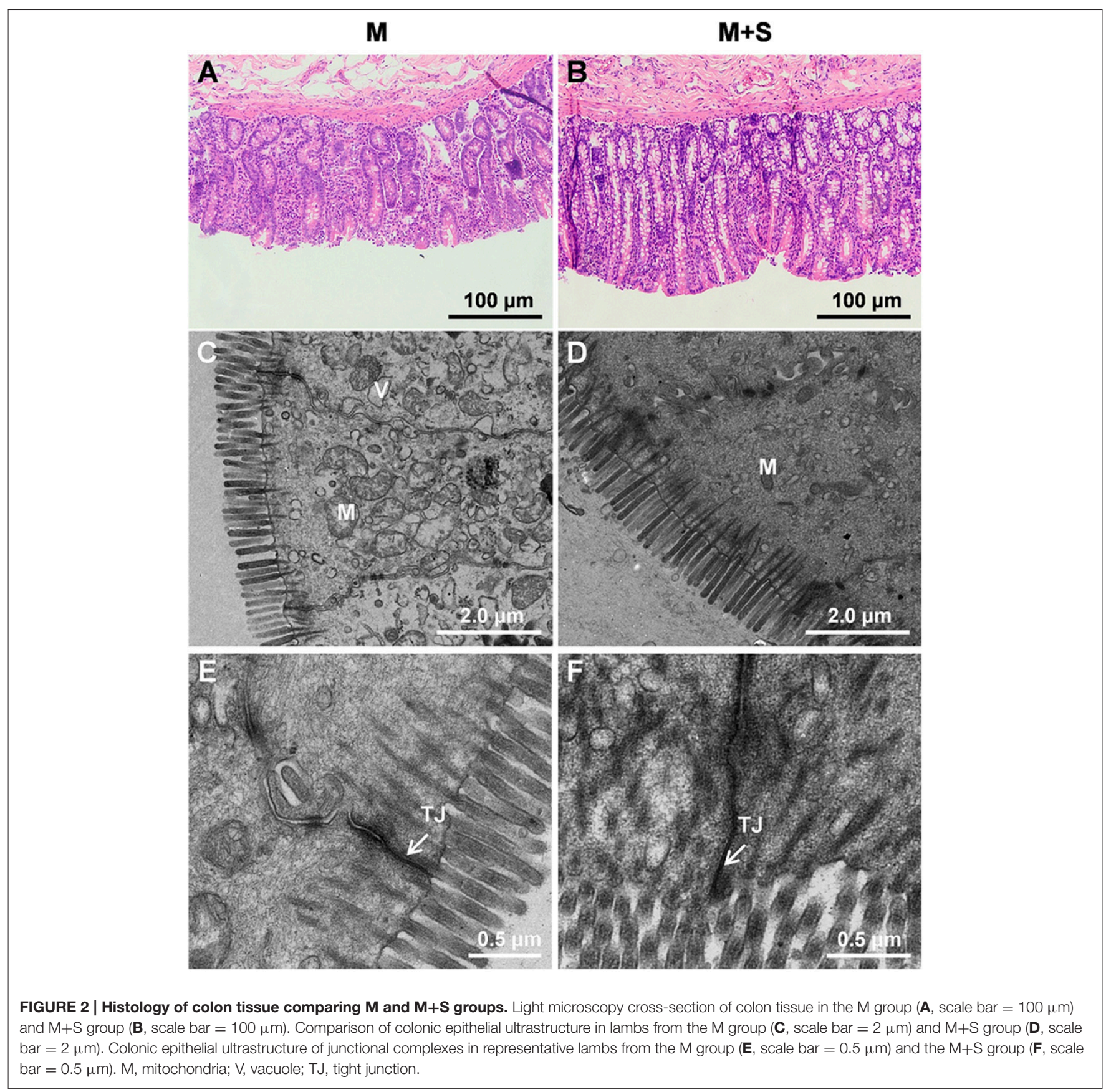

( $P=0.0017)$, Parabacteroides $(P=0.0083)$, and Ruminobacter $(P$ $=0.0080)$.

\section{DISCUSSION}

Colonic mucosal microbiota play important roles in host metabolism and immune homeostasis, thus affecting the health of ruminants. Early nutritional intervention is an advantageous strategy for modulating gastrointestinal microbiota, and their development profile can impact host health. In the current study, we found that supplementation of breast milk with concentrate starter feeding can regulate colonic mucosal bacterial composition and structure, and that these changes were associated with variations in the mRNA expression of TLR and cytokines. These findings may provide new insights into colonic mucosal bacteria and immune homeostasis in developing lambs.

The similar final body weight gain in the $\mathrm{M}$ and $\mathrm{M}+\mathrm{S}$ groups suggests that lambs in the two groups had a similar total nutrient intake. We found that concentrate starter feeding increased the bacterial richness of the colonic mucosal community as reflected by a higher Chao 1 value, which is somewhat inconsistent with findings on the colonic mucosal community of concentrate-fed 

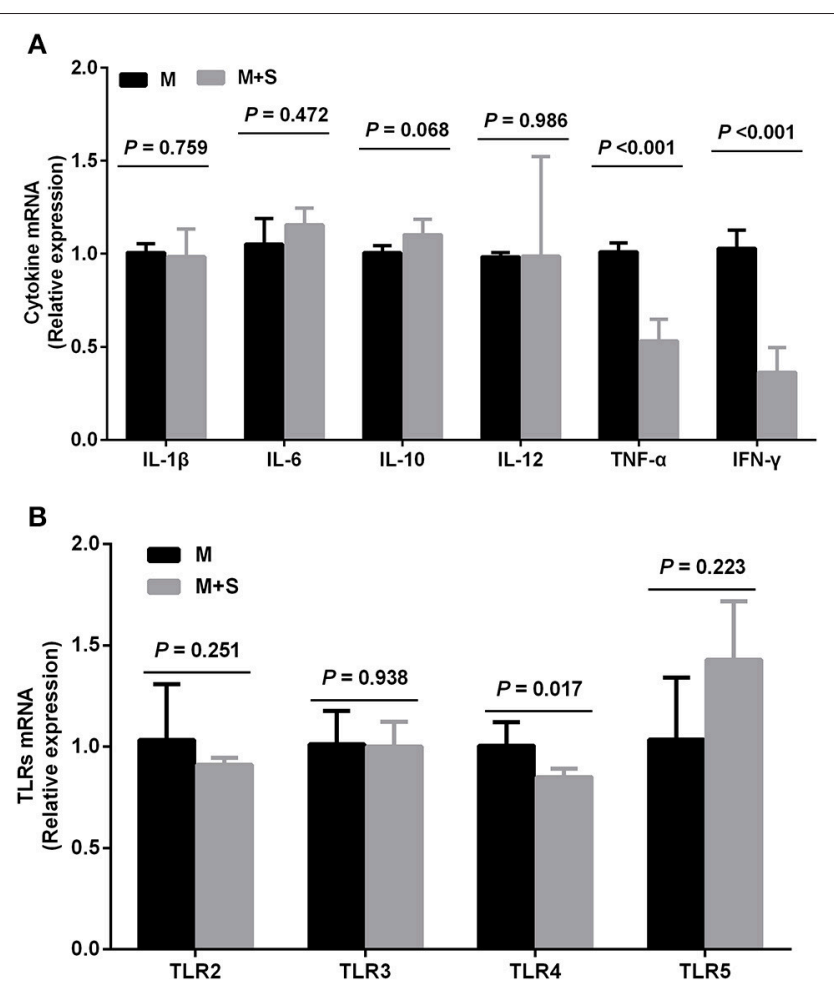

FIGURE 3 | Changes in the relative mRNA expression of cytokines (A) and TLR (B) in the colonic mucosa of lambs during starter feeding (means \pm $S D, n=8)$. The relative amount of each was normalized to GAPDH mRNA levels as a housekeeping gene, and the data were analyzed according to the $2^{-\Delta \Delta C T}$ method.

adult goats (Ye et al., 2016). Moreover, the PCA and AMOVA analyses showed that concentrate starter feeding significantly affected colonic mucosal bacterial composition and structure. In this study, concentrate (especially starch) was supplemented in $\mathrm{M}+\mathrm{S}$ lambs, but not $\mathrm{M}$ lambs, which resulted in the most remarkable difference between these two feeding strategies. Thus, more amount of starch substrates may flow into the colon of $\mathrm{M}+\mathrm{S}$ lambs. As expected, we also found that $\mathrm{M}+\mathrm{S}$ lambs had lower colonic $\mathrm{pH}$ and higher colonic VFA and lactate concentrations. Therefore, the changes in colonic luminal environment may contribute to the changes in colonic mucosal bacterial composition and structure.

At the phylum level, we found that Firmicutes, Bacteroidetes, and Proteobacteria were the dominant phyla associated with the colonic mucosa of lambs, which agrees with data found for preweaned calves (Malmuthuge et al., 2014) and goat kids (Jiao et al., 2015b). Meanwhile, compared with lambs in the M group, lambs in the $\mathrm{M}+\mathrm{S}$ group had a lower relative abundance of Firmicutes and a higher proportion of Bacteroidetes. Similar results were observed in the colonic mucosa of high-concentrate diet-fed goats (Ye et al., 2016) and in the colonic digesta of concentrate-fed goat kids (Jiao et al., 2016).

At the genus level, starter feeding increased the relative abundances of unclassified S24-7 (family), Prevotella, Ruminobacter, Oscillibacter, Parabacteroides, and
Bifidobacterium, but decreased the proportions of unclassified Ruminococcaceae (family), Blautia, Campylobacter, Butyrivibrio, Pseudobutyrivibrio, and Lactobacillus. On the one hand, the enrichment of starch degraders, like unclassified S24-7, Prevotella, Bifidobacterium, and Ruminobacter, may be due to greater starch availability in the colon during starter feeding. Other studies have demonstrated the presence of family S24-7 in dairy and beef cattle (McCann et al., 2014; Lima et al., 2015; Anderson et al., 2016); however, the role of S24-7 in the colon of ruminants remains poorly understood. Bacteria belonging to family S24-7 have also been identified in the colons of mice fed high-fat diets and gluco-oligosaccharides (Serino et al., 2012). Therefore, it is possible that the family S24-7 is capable of starch utilization (Serino et al., 2012; Anderson et al., 2016). As expected, concentrate starter feeding increased the proportion of Prevotella (a kind of starch degrader) in the colonic mucosa of lambs. Similarly, previous studies have demonstrated that high-grain diet feeding increases the abundance of Prevotella in the colons of adult goats (Metzler-Zebeli et al., 2013; Ye et al., 2016) and goat kids (Jiao et al., 2016). Bifidobacterium, a starch-hydrolyzing bacteria, can produce acetate and lactate fermentation end products (Xia et al., 2015). Other studies have found a higher abundance of Bifidobacterium in the rumen of high-concentrate-fed calves (Trovatelli and Matteuzzi, 1976) and dairy cows (Zened et al., 2013). This result also partly explains why the starter-fed lambs in our study had higher lactate concentrations in their colons. Additionally, the genus Ruminobacter is also involved in starch degradation (Halbrügge and Walter, 1990; Anderson, 1995). On the other hand, Ruminococcaceae and members of the Lachnospiraceae family are important fibrolytic bacteria in the guts of mammals (Biddle et al., 2013; Li et al., 2014). Thus, lower fibrous substrate availability in the colon may have contributed to a decrease in fibrolytic bacteria (unclassified Ruminococcaceae, Blautia, Butyrivibrio, and Pseudobutyrivibrio) in the $\mathrm{M}+\mathrm{S}$ group.

Furthermore, changes in colonic mucosal bacterial composition may partly impact host immune homeostasis in the colon, and dysregulated immune responses to opportunistic commensals potentially affect host health (Donaldson et al., 2016). In the current study, we found that starter feeding increased the relative abundances of Oscillibacter, Parabacteroides, and Bifidobacterium, but decreased the proportions of unclassified Ruminococcaceae, Blautia, and Campylobacter in the colonic mucosa of lambs. Among these variation taxa, Oscillibacter is a bacteria found in the colonic mucosa of humans. Reports have shown that healthy people have a higher abundance of Oscillibacter in their colonic mucosa than patients diagnosed with Crohn's disease (Man et al., 2011; Mondot et al., 2011), which indicates that Oscillibacter may be beneficial for colonic health. Some species of Parabacteroides significantly reduce the severity of intestinal inflammation in murine models of acute and chronic colitis induced by dextran sulfate sodium (Kverka et al., 2011). Some species of Bifidobacterium, which produce acetate and lactate, are beneficial to the colonic health of both animals and humans (Gibson et al., 2004) and to the normalization of the ratio of anti-inflammatory to proinflammatory cytokines (O’Mahony et al., 2005). Thus, 


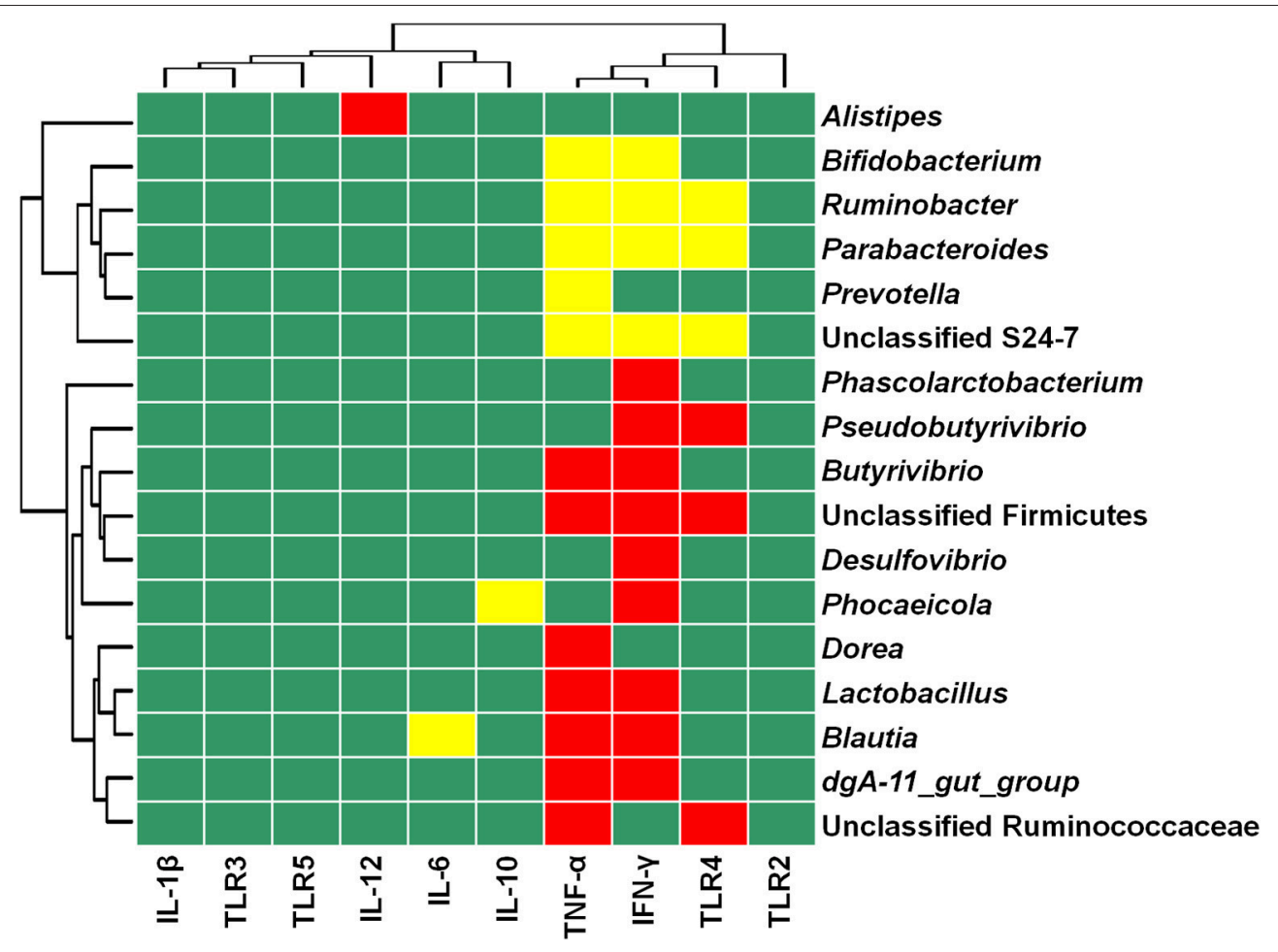

FIGURE 4 | Heat map showing the correlation between the relative abundances of bacterial taxa and mRNA expression in colonic mucosa. The top 50 bacterial taxa were selected to perform the correlation analyses, and those significantly associated with TLR and cytokines are shown. Cells are colored based on Spearman's correlation coefficient. Red represents a significant positive correlation $(P<0.01)$, yellow represents a significant negative correlation $(P<0.01)$, and green represents a non-significant correlation $(P>0.01)$.

our findings indicate that the higher relative abundances of some beneficial bacteria (Oscillibacter, Parabacteroides, and Bifidobacterium) during concentrate starter feeding may have beneficial effects on the colonic health of lambs in the milk-feeding period.

Ruminococcaceae is the dominant family in the colonic mucosa of mammals and has been associated with the maintenance of gut health (Donaldson et al., 2016). Previous studies have shown that the enrichment of this family is associated with colonic mucosal inflammation (Willing et al., 2010). Moreover, the enrichment of Blautia has been related to colonic inflammation in humans (Loh and Blaut, 2012). Thus, starter feeding-induced depression of Blautia may be beneficial for alleviating local inflammation in lamb colons. Our recent study showed that high-grain diet feeding increases the relative abundance of Blautia in the colonic mucosa of adult goats (Ye et al., 2016). This discrepancy indicates that colonic mucosal bacteria reflect different responses to concentrate diet feeding in preweaned and adult ruminants. Some studies have found Campylobacter in the colons of cattle (Inglis et al., 2005), goat kids (Jiao et al., 2016), and sheep (Stanley and Jones, 2003), while other studies have demonstrated that the enrichment of some specific species of Campylobacter is closely associated with local inflammation in the colons of humans and animals (Russell et al., 1993; Chen et al., 2006). Thus, these findings suggest that starter feeding-induced depression in the proportion of some pathenogens and potential pathenogens (unclassified Ruminococcaceae, Blautia, and Campylobacter) may also have beneficial effects on lamb health.

Colonic mucosal microbiota are integral for stimulating the innate immune response of the host (Abreu, 2010). In the current study, we found that concentrate starter feeding decreased TLR4 expression, which agrees with Jiao et al. (2016), who demonstrated that supplemental feeding (compared with grazing) decreases TLR4 expression. TLR4 can recognize Gram-negative bacteria and their products (Abreu, 2010). Surprisingly, the correlation analysis revealed that TLR4 expression is positively associated with some Gram-positive bacterial taxa (unclassified Ruminococcaceae, Pseudobutyrivibrio, and unclassified Firmicutes) and negatively associated with some Gram-negative bacterial taxa (unclassified S24-7, Parabacteroides, and Ruminobacter) in the colonic mucosa of lambs. The changes in Gram-negative bacterial products during starter feeding may contribute to this discrepancy.

It has been reported that TLR can recognize some specific commensal bacteria and their products and then initiate proinflammatory pathways (Abreu, 2010). Thus, the effect of starter feeding on proinflammatory cytokine expression was also investigated. The data show that concentrate starter feeding decreased mRNA expression of the cytokines TNF- $\alpha$ 
and IFN- $\gamma$ in the colonic tissue of lambs. These results were somewhat consistent with Jiao et al. (2016), who indicated that supplemental feeding (compared with grazing) decreased IL-6 expression. The correlation analysis further revealed that the depression of mRNA expression in cytokines is associated with some specific bacteria. In particular, TNF$\alpha$ and IFN $-\gamma$ are negatively correlated with Parabacteroides and Bifidobacterium, respectively, and positively associated with Blautia and unclassified Ruminococcaceae, respectively. As mentioned earlier, treatment with Parabacteroides prevented dextran sodium sulfate-induced increases in proinflammatory cytokines IL-6 and IFN- $\gamma$ in mice colons (Kverka et al., 2011). Some species of Bifidobacterium are considered beneficial to the colonic health of animals and humans (Gibson et al., 2004). On the other hand, Blautia is related to colonic mucosal inflammation in humans (Loh and Blaut, 2012). Previous studies have also shown that the enrichment of the Ruminococcaceae family is associated with colonic mucosal inflammation (Willing et al., 2010). A high-fat, dietinduced increase of proinflammatory cytokine (IL-1 $\beta$, IL-6, and TNF- $\alpha$ ) expression has been associated with the enrichment of Ruminococcaceae in the colonic tissue of mice (Kim et al., 2012). Thus, the decreased expression of cytokines in our study may be partly due to enrichments of some beneficial bacteria (Parabacteroides and Bifidobacterium) and the depression of some pathenogens and potential pathenogens (Blautia and Ruminococcaceae family) during starter feeding in lambs. Our findings show that starter feeding increased the abundance of some beneficial bacteria while decreasing the proportion of some pathenogens and potential pathenogens, which could in turn protect colonic mucosal morphology and modulate immune homeostasis in preweaned lambs. Certainly, these starter feeding-induced responses may not be necessarily beneficial for postweaning health in ruminants. Many previous studies indicated that the upregulation of TLR and cytokine genes to a certain degree may faciliate gastrointestinal immune system development (Abreu, 2010; Chen et al., 2012). It is possible that the increase in TLR4, TNF- $\alpha$, and IFN- $\gamma$ levels in breast-milk-fed lambs are actually beneficial to the developing immune system and that the starter feeding could contribute to problems observed in later life. Thus, more studies are needed to investigate whether starter feeding affects postweaning health in ruminants.

\section{REFERENCES}

Abecia, L., Martín-Garcia, A. I., Martínez, G., Newbold, C. J., and Yáñez-Ruiz, D. R. (2013). Nutritional intervention in early life to manipulate rumen microbial colonization and methane output by kid goats postweaning. J. Anim. Sci. 91, 4832-4840. doi: 10.2527/jas.2012-6142

Abreu, M. T. (2010). Toll-like receptor signalling in the intestinal epithelium: how bacterial recognition shapes intestinal function. Nat. Rev. Immunol. 10, 131-144. doi: 10.1038/nri2707

Anderson, C. L., Schneider, C. J., Erickson, G. E., MacDonald, J. C., and Fernando, S. C. (2016). Rumen bacterial communities can be acclimated faster to high concentrate diets than currently implemented feedlot programs. J. Appl. Microbiol. 120, 588-599. doi: 10.1111/jam.13039

\section{CONCLUSION}

We found that concentrate starter feeding increased colonic fermentation and significantly affected colonic mucosal bacterial communities by increasing the relative abundances of the dominant taxa unclassified S24-7, Oscillibacter, Prevotella, Parabacteroides, Bifidobacterium, Ruminobacter, and Succinivibrio, and decreasing the proportions of unclassified Ruminococcaceae, RC9_gut_group, Blautia, Phocaeicola, Phascolarctobacterium, unclassified BS11_gut_group, unclassified family_XIII, Campylobacter, unclassified Firmicutes, Pseudobutyrivibrio, Barnesiella, Lactobacillus, unclassified Gastranaerophilales, Butyrivibrio, dgA-11_gut_group, and Dorea in lambs. Meanwhile, starter feeding decreased the colonic mucosal mRNA expression of TLR4 and cytokines TNF- $\alpha$ and IFN- $\gamma$. Furthermore, the changes in mRNA expression of TLR and cytokines were associated with variations in the abundances of some specific bacteria in colonic mucosa. Collectively, our study shows that concentrate starter feeding can alter colonic mucosal bacterial composition and modulate mucosal immune homeostasis during the milk-feeding period in lambs.

\section{AUTHOR CONTRIBUTIONS}

DS and JL carried out the majority of the experiment including animal care, VFA analyses, RNA isolation, and real-time PCR. GB and JL were responsible for pyrosequencing data processing, analyses and interpretation. JL, SM, and WZ contributed to the conception of the project. The manuscript was prepared by JL and SM.

\section{ACKNOWLEDGMENTS}

This work was supported by the National Natural Science Foundation of China (31501980), Fundamental Research Funds for the Central Universities (KJQN201610), and the China Postdoctoral Science Foundation (2016T90474).

\section{SUPPLEMENTARY MATERIAL}

The Supplementary Material for this article can be found online at: http://journal.frontiersin.org/article/10.3389/fmicb. 2017.00429/full\#supplementary-material

Anderson, K. L. (1995). Biochemical analysis of starch degradation by Ruminobacter amylophilus 70. Appl. Environ. Microbiol. 61, 1488-1491.

Barker, S. B., and Summerson, W. H. (1941). The colorimetric determination of lactic acid in biological material. J. Biol. Chem. 138, 535-554.

Biddle, A., Stewart, L., Blanchard, J., and Leschine, S. (2013). Untangling the genetic basis of fibrolytic specialization by Lachnospiraceae and Ruminococcaceae in diverse gut communities. Diversity 5:627. doi: 10.3390/d5030627

Caporaso, J. G., Bittinger, K., Bushman, F. D., DeSantis, T. Z., Andersen, G. L., and Knight, R. (2010a). PyNAST: a flexible tool for aligning sequences to a template alignment. Bioinformatics 26, 266-267. doi: 10.1093/bioinformatics/btp636

Caporaso, J. G., Kuczynski, J., Stombaugh, J., Bittinger, K., Bushman, F. D., Costello, E. K., et al. (2010b). QIIME allows analysis of 
high-throughput community sequencing data. Nat. Methods 7, 335-336. doi: 10.1038/nmeth.f.303

Charavaryamath, C., Fries, P., Gomis, S., Bell, C., Doig, K., Guan, L. L., et al. (2011). Mucosal changes in a long-term bovine intestinal segment model following removal of ingesta and microflora. Gut Microbes 2, 134-144. doi: 10.4161/gmic.2.3.16483

Chen, M. L., Ge, Z., Fox, J. G., and Schauer, D. B. (2006). Disruption of tight junctions and induction of proinflammatory cytokine responses in colonic epithelial cells by Campylobacter jejuni. Infect. Immun. 74, 6581-6589. doi: 10.1128/IAI.00958-06

Chen, Y., Oba, M., and Guan, L. L. (2012). Variation of bacterial communities and expression of Toll-like receptor genes in the rumen of steers differing in susceptibility to subacute ruminal acidosis. Vet. Microbiol. 159, 451-459. doi: $10.1016 /$ j.vetmic.2012.04.032

Chomczynski, P., and Sacchi, N. (1987). Single-step method of RNA isolation by acid guanidinium thiocyanate-phenol-chloroform extraction. Anal. Biochem. 162, 156-159. doi: 10.1016/0003-2697(87)90021-2

DeSantis, T. Z., Hugenholtz, P., Larsen, N., Rojas, M., Brodie, E. L., Keller, K., et al. (2006). Greengenes, a chimera-checked 16S rRNA gene database and workbench compatible with ARB. Appl. Environ. Microbiol. 72, 5069-5072. doi: 10.1128/AEM.03006-05

Donaldson, G. P., Lee, S. M., and Mazmanian, S. K. (2016). Gut biogeography of the bacterial microbiota. Nat. Rev. Microbiol. 14, 20-32. doi: 10.1038/nrmicro3552

Edgar, R. C. (2013). UPARSE: highly accurate OTU sequences from microbial amplicon reads. Nat. Methods 10:996. doi: 10.1038/nmeth.2604

Edgar, R. C., Haas, B. J., Clemente, J. C., Quince, C., and Knight, R. (2011). UCHIME improves sensitivity and speed of chimera detection. Bioinformatics 27, 2194-2200. doi: 10.1093/bioinformatics/btr381

Gibson, G. R., Probert, H. M., Loo, J. V., Rastall, R. A., and Roberfroid, M. B. (2004). Dietary modulation of the human colonic microbiota: updating the concept of prebiotics. Nutr. Res. Rev. 17, 259-275. doi: 10.1079/nrr 200479

Halbrügge, M., and Walter, U. (1990). Analysis, purification and properties of a 50,000-dalton membrane-associated phosphoprotein from human platelets. J. Chromatogr. 521, 335-343. doi: 10.1016/0021-9673(90)85057-3

Inglis, G. D., Kalischuk, L. D., Busz, H. W., and Kastelic, J. P. (2005). Colonization of cattle intestines by Campylobacter jejuni and Campylobacter lanienae. Appl. Environ. Microbiol. 71, 5145-5153. doi: 10.1128/AEM.71.9.5145-5153.2005

Jiao, J. Z., Li, X. P., Beauchemin, K. A., Tan, Z. L., Tang, S. X., and Zhou, C. S. (2015a). Rumen development process in goats as affected by supplemental feeding v. grazing: age-related anatomic development, functional achievement and microbial colonisation. Brit. J. Nutr. 113, 888-900. doi: 10.1017/S0007114514004413

Jiao, J. Z., W, Z., Guan, L. L., Tan, Z. L., Han, X. F., Tang, S. X., et al. (2015b). Postnatal bacterial succession and functional establishment of hindgut in supplemental feeding and grazing goats. J. Anim. Sci. 93, 3528-3538. doi: $10.2527 /$ jas.2014-8706

Jiao, J., Lu, Q., Forster, R. J., Zhou, C., Wang, M., Kang, J., et al. (2016). Age and feeding system (supplemental feeding versus grazing) modulates colonic bacterial succession and host mucosal immune maturation in goats. J. Anim. Sci. 94, 2506-2518. doi: 10.2527/jas.2015-0081

Kim, K. A., Gu, W., Lee, I. A., Joh, E. H., and Kim, D. H. (2012). High fat diet-induced gut microbiota exacerbates inflammation and obesity in mice via the TLR4 signaling pathway. PLoS ONE 7:e47713. doi: 10.1371/journal.pone.0047713

Kverka, M., Zakostelska, Z., Klimesova, K., Sokol, D., Hudcovic, T., Hrncir, T., et al. (2011). Oral administration of Parabacteroides distasonis antigens attenuates experimental murine colitis through modulation of immunity and microbiota composition. Clin. Exp. Immunol. 163, 250-259. doi: 10.1111/j.1365-2249.2010.04286.x

Li, D., Leahy, S., Henderson, G., Kelly, W., Cookson, A., Attwood, G., et al. (2014). Atypical bacterial rRNA operon structure is prevalent within the Lachnospiraceae, and use of the 16S-23S rRNA internal transcribed spacer region for the rapid identification of ruminal Butyrivibrio and Pseudobutyrivibrio strains. Ann. Microbiol. 64, 1623-1631. doi: 10.1007/s13213-014-0806-2

Lima, F. S., Oikonomou, G., Lima, S. F., Bicalho, M. L., Ganda, E. K., Filho, J. C., et al. (2015). Prepartum and postpartum rumen fluid microbiomes: characterization and correlation with production traits in dairy cows. Appl. Environ. Microbiol. 81, 1327-1337. doi: 10.1128/aem.03138-14

Liu, J. H., Bian, G. R., Sun, D. M., Zhu, W. Y., and Mao, S. Y. (2017). Starter feeding altered ruminal epithelial bacterial communities and some key immunerelated genes' expression before weaning in lambs. J. Anim. Sci. 95, 910-921. doi: $10.2527 /$ jas.2016.0985

Liu, J. H., Bian, G. R., Zhu, W. Y., and Mao, S. Y. (2015). High-grain feeding causes strong shifts in ruminal epithelial bacterial community and expression of Toll-like receptor genes in goats. Front. Microbiol. 6:167. doi: 10.3389/fmicb.2015.00167

Liu, J. H., Xu, T. T., Liu, Y. J., Zhu, W. Y., and Mao, S. Y. (2013). A highgrain diet causes massive disruption of ruminal epithelial tight junctions in goats. Am. J. Physiol. Regul. Integr. Comp. Physiol. 305, R232-R241. doi: 10.1152/ajpregu.00068.2013

Livak, K. J., and Schmittgen, T. D. (2001). Analysis of relative gene expression data using real-time quantitative PCR and the $2^{-\Delta \Delta C T}$ method. Methods 25, 402-408. doi: 10.1006/meth.2001.1262

Loh, G., and Blaut, M. (2012). Role of commensal gut bacteria in inflammatory bowel diseases. Gut Microbes 3, 544-555. doi: 10.4161/gmic. 22156

Lozupone, C., and Knight, R. (2005). UniFrac: a new phylogenetic method for comparing microbial communities. Appl. Environ. Microb. 71, 8228-8235. doi: 10.1128/AEM.71.12.8228-8235.2005

Malmuthuge, N., Griebel, P. J., and Guan le, L. (2014). Taxonomic identification of commensal bacteria associated with the mucosa and digesta throughout the gastrointestinal tracts of preweaned calves. Appl. Environ. Microbiol. 80, 2021-2028. doi: 10.1128/AEM.03864-13

Malmuthuge, N., Li, M., Goonewardene, L. A., Oba, M., and Guan, L. L. (2013). Effect of calf starter feeding on gut microbial diversity and expression of genes involved in host immune responses and tight junctions in dairy calves during weaning transition. J. Dairy Sci. 96, 3189-3200. doi: 10.3168/jds. 2012-6200

Man, S. M., Kaakoush, N. O., and Mitchell, H. M. (2011). The role of bacteria and pattern-recognition receptors in Crohn's disease. Nat. Rev. Gastro. Hepat. 8, 152-168. doi: 10.1038/nrgastro.2011.3

Mao, S. Y., Zhang, M. L., Liu, J. H., and Zhu, W. Y. (2015). Characterising the bacterial microbiota across the gastrointestinal tracts of dairy cattle: membership and potential function. Sci. Rep. 5:16116. doi: 10.1038/srep16116

McCann, J. C., Drewery, M. L., Sawyer, J. E., Pinchak, W. E., and Wickersham, T. A. (2014). Effect of postextraction algal residue supplementation on the ruminal microbiome of steers consuming low-quality forage. J. Anim. Sci. 92, 5063-5075. doi: 10.2527/jas.2014-7811

Metzler-Zebeli, B. U., Schmitz-Esser, S., Klevenhusen, F., Podstatzky-Lichtenstein, L., Wagner, M., and Zebeli, Q. (2013). Grain-rich diets differently alter ruminal and colonic abundance of microbial populations and lipopolysaccharide in goats. Anaerobe 20, 65-73. doi: 10.1016/j.anaerobe.2013.02.005

Ministry of Agriculture of China (2004). Feeding Standard of Meat Producing Sheep and Goats. Report number NY/T816-2004. Minist. Agric. Beijing.

Mondot, S., Kang, S., Furet, J. P., de Carcer, D. A., McSweeney, C., Morrison, M., et al. (2011). Highlighting new phylogenetic specificities of Crohn's disease microbiota. Inflamm. Bowel Dis. 17, 185-192. doi: 10.1002/ibd.21436

O’Mahony, L., McCarthy, J., Kelly, P., Hurley, G., Luo, F., Chen, K., et al. (2005). Lactobacillus and Bifidobacterium in irritable bowel syndrome: symptom responses and relationship to cytokine profiles. Gastroenterology 128, 541-551. doi: 10.1053/j.gastro.2004.11.050

Qin, W. L. (1982). Determination of rumen volatile fatty acids by means of gas chromatography. J. Nj. Agric. Coll. 4, 110-116.

Russell, R. G., O’Donnoghue, M., Blake, D. C. Jr., Zulty, J., and DeTolla, L. J. (1993). Early colonic damage and invasion of Campylobacter jejuni in experimentally challenged infant Macaca mulatta. J. Infect. Dis. 168, 210-215. doi: 10.1093/infdis/168.1.210

Schloss, P. D., Westcott, S. L., Ryabin, T., Hall, J. R., Hartmann, M., Hollister, E. B., et al. (2009). Introducing mothur: open-source, platformindependent, community-supported software for describing and comparing microbial communities. Appl. Environ. Microbiol. 75, 7537-7541. doi: 10.1128/aem.01541-09

Serino, M., Luche, E., Gres, S., Baylac, A., Bergé, M., Cenac, C., et al. (2012). Metabolic adaptation to a high-fat diet is associated with a change in the gut microbiota. Gut 61, 543-553. doi: 10.1136/gutjnl-2011-301012 
Stanley, K., and Jones, K. (2003). Cattle and sheep farms as reservoirs of Campylobacter. J. Appl. Microbiol. 94, 104S-113S. doi: 10.1046/j.1365-2672.94.s1.12.x

Steele, M. A., Croom, J., Kahler, M., AlZahal, O., Hook, S. E., Plaizier, K., et al. (2011). Bovine rumen epithelium undergoes rapid structural adaptations during grain-induced subacute ruminal acidosis. Am. J. Physiol. Regul. Integr. Comp. Physiol. 300, R1515-R1523. doi: 10.1152/ajpregu. 00120.2010

Trovatelli, L. D., and Matteuzzi, D. (1976). Presence of bifidobacteria in the rumen of calves fed different rations. Appl. Environ. Microbiol. 32, 470-473.

Wang, A., Gu, Z., Heid, B., Akers, R. M., and Jiang, H. (2009). Identification and characterization of the bovine G protein-coupled receptor GPR41 and GPR43 genes. J. Dairy Sci. 92, 2696-2705. doi: 10.3168/jds.2009-2037

Wang, Q., Garrity, G. M., Tiedje, J. M., and Cole, J. R. (2007). Naive Bayesian classifier for rapid assignment of rRNA sequences into the new bacterial taxonomy. Appl. Environ. Microbiol. 73, 5261-5267. doi: 10.1128/AEM.00062-07

Wang, W., Li, C., Li, F., Wang, X., Zhang, X., Liu, T., et al. (2016). Effects of early feeding on the host rumen transcriptome and bacterial diversity in lambs. Sci. Rep. 6:32479. doi: 10.1038/srep32479

Willing, B. P., Dicksved, J., Halfvarson, J., Andersson, A. F., Lucio, M., Zheng, Z., et al. (2010). A pyrosequencing study in twins shows that gastrointestinal microbial profiles vary with inflammatory bowel disease phenotypes. Gastroenterology 139, 1844-1854. doi: 10.1053/j.gastro.2010. 08.049
Xia, Y., Kong, Y., Seviour, R., Yang, H. E., Forster, R., Vasanthan, T., et al. (2015). In situ identification and quantification of starch-hydrolyzing bacteria attached to barley and corn grain in the rumen of cows fed barley-based diets. FEMS Microbiol. Ecol. 91:fiv077. doi: 10.1093/femsec/fiv077

Yáñez-Ruiz, D. R., Abecia, L., and Newbold, C. J. (2015). Manipulating rumen microbiome and fermentation through interventions during early life: a review. Front. Microbiol. 6:1133. doi: 10.3389/fmicb.2015.01133

Ye, H., Liu, J., Feng, P., Zhu, W., and Mao, S. (2016). Grain-rich diets altered the colonic fermentation and mucosa-associated bacterial communities and induced mucosal injuries in goats. Sci. Rep. 6:20329. doi: 10.1038/srep20329

Zened, A., Combes, S., Cauquil, L., Mariette, J., Klopp, C., Bouchez, O., et al. (2013). Microbial ecology of the rumen evaluated by 454 GS FLX pyrosequencing is affected by starch and oil supplementation of diets. FEMS Microbiol. Ecol. 83, 504-514. doi: 10.1111/1574-6941.12011

Conflict of Interest Statement: The authors declare that the research was conducted in the absence of any commercial or financial relationships that could be construed as a potential conflict of interest.

Copyright (c) 2017 Liu, Bian, Sun, Zhu and Mao. This is an open-access article distributed under the terms of the Creative Commons Attribution License (CC BY). The use, distribution or reproduction in other forums is permitted, provided the original author(s) or licensor are credited and that the original publication in this journal is cited, in accordance with accepted academic practice. No use, distribution or reproduction is permitted which does not comply with these terms. 